\title{
O PAPEL ATUAL DA ULTRA-SONOGRAFIA TRANSRETAL DA PRóstata NA DETECC̣Ão PRECOCE do CÂNCER PROSTÁtICO*
}

\author{
Viviane Cristine Tavares Santos ${ }^{1}$, Miguel Angelo Milito ${ }^{2}$, Edson Marchiori ${ }^{3}$
}

Resumo OBJETIVO: Determinar o papel real que a ultra-sonografia da próstata, notadamente com a associação do Doppler colorido, desempenha no diagnóstico de lesões malignas na próstata. MATERIAIS E MÉTODOS: Foram estudados, prospectivamente, 84 pacientes submetidos a biópsia guiada por ultra-sonografia transretal. Em todos os pacientes foram feitos estudo com Doppler colorido, à procura de focos de hipervascularização, e o exame ultra-sonográfico habitual. Os resultados foram comparados com os diagnósticos histopatológicos obtidos. RESULTADOS: A ultra-sonografia transretal habitual (escala de cinza) apresentou sensibilidade de $67,7 \%$, especificidade de $52,8 \%$, valor preditivo positivo de $45,6 \%$ e valor preditivo negativo de $73,6 \%$. A adição do estudo com Doppler colorido ocasionou aumento importante da especificidade (de $52,8 \%$ para $79,2 \%$ ) e do valor preditivo positivo (de $45,6 \%$ para $62,0 \%$ ), porém causou queda na sensibilidade (de $67,7 \%$ para $58,0 \%$ ). Além disso, houve perda de $32,2 \%$ dos cânceres, que não foram diagnosticados por nenhum dos dois métodos, e esses pacientes, apesar de possuírem cânceres menos extensos, eram todos clinicamente significativos (Gleason 6 ou mais). CONCLUSÃO: Mesmo com a associação do Doppler colorido, a ultra-sonografia transretal não possui capacidade suficiente para definir, através dos seus achados, quais pacientes devem ou não realizar biópsia.

Unitermos: Próstata; Câncer prostático; Ultra-sonografia transretal; Doppler colorido; Biópsia prostática.

Abstract Current role of transrectal ultrasonography in the early detection of prostate cancer.

OBJECTIVE: To determine the role of transrectal ultrasound, especially in association with color Doppler, in the diagnosis of prostate cancer. MATERIALS AND METHODS: Eighty-four cases of patients who underwent transrectal biopsy were prospectively analyzed. In all patients, besides the traditional gray-scale sonogram, color Doppler study was performed in search of hypervascular foci. The results were compared with the histopathologic diagnosis. RESULTS: The gray-scale sonogram showed a $67.7 \%$ sensitivity, $52.8 \%$ specificity, positive predictive value of $45.6 \%$ and negative predictive value of $73.6 \%$. The association with color Doppler increased the specificity (from $52.8 \%$ to $\mathbf{7 9 . 2} \%$ ) and the positive predictive value (from $45.6 \%$ to $62.0 \%$ ) although it has caused a decrease in the sensitivity (from $67.7 \%$ to $58.0 \%$ ). Besides, the study had $32.2 \%$ of missed cancers by both methods, and these patients, although having less extensive cancers all of them were clinically significant (Gleason 6 or more). CONCLUSION: Transrectal ultrasound findings, even in association with color Doppler, are not sufficiently accurate to determine whether a patient should or not be submitted to biopsy.

Keywords: Prostate; Prostatic cancer; Transrectal ultrasound; Color Doppler; Prostate biopsy.

\section{INTRODUÇÃO}

O câncer de próstata é, atualmente, um problema de saúde mundial. No Brasil, tornou-se um problema de saúde pública,

* Trabalho realizado no Serviço de Radiologia e Radiodiagnóstico do Hospital Universitário Clementino Fraga Filho da Universidade Federal do Rio de Janeiro, Rio de Janeiro, RJ.

1. Médica Radiologista, Mestranda do Departamento de Radiologia da Faculdade de Medicina da Universidade Federal do Rio de Janeiro.

2. Médico Assistente do Serviço de Radiologia e Radiodiagnóstico do Hospital Universitário Clementino Fraga Filho.

3. Professor Titular de Radiologia da Universidade Federal Fluminense, Coordenador Adjunto do Curso de Pós-Graduação em Radiologia da Universidade Federal do Rio de Janeiro.

Endereço para correspondência: Dra. Viviane Cristine Tavares Santos. Rua Gonzaga Bastos, 123/302, Vila Isabel. Rio de Janeiro, RJ, 20541-000. E-mail: vivianecristine@ibest.com.br; vivisantos@ig.com.br

Recebido para publicação em 4/3/2005. Aceito, após revisão, em 3/8/2005. uma vez que representa o segundo câncer mais comum em homens e apresenta tendência de aumento nos últimos anos, devido ao envelhecimento da população ${ }^{(\mathbf{1})}$.

Por esse motivo, o carcinoma prostático necessita de ferramentas de detecção precisas o suficiente para promover sua detecção precoce, permitindo, assim, o tratamento mais adequado, melhorando a sobrevida e diminuindo a morbidade. Atualmente, o diagnóstico do câncer de próstata se baseia no toque retal, nos níveis sanguíneos do antígeno prostático específico (PSA) e na ultra-sonografia transretal, porém nenhum deles é sensível e específico o suficiente para ser usado sozinho na definição da conduta a ser tomada em relação ao paciente $e^{(2,3)}$.
O PSA é ferramenta diagnóstica simples, muito usada no rastreio do câncer de próstata, porém pode ter significado dúbio quando seu aumento for intermediário. A maior utilidade do PSA é como indicador de volume tumoral e progressão da doença após tratamento inicial ${ }^{(\mathbf{4 , 5})}$.

A ultra-sonografia transretal é um método que, apesar de não possuir a sensibilidade e a especificidade que se acreditava ter no início de seu desenvolvimento, consegue detectar maior quantidade de tumores e em estágios mais precoces do que os outros métodos. Além disso, ocorreram avanços técnicos como a introdução do Doppler colorido, que apesar de não haver correspondido plenamente às expectativas iniciais, é um fator adjuvante importante 
na procura do câncer prostático, ao aumentar o valor preditivo positivo e a sensibilidade $^{(2,6,7)}$.

Associado a esses avanços, o uso da ultra-sonografia foi bem estabelecido como método principal para orientar a colheita de material por meio de biópsias prostáticas, de maneira rápida, segura e relativamente indolor $^{(6,7)}$.

Os objetivos deste trabalho foram determinar a sensibilidade, a especificidade, os valores preditivos positivo e negativo da ultra-sonografia transretal, tanto da escala de cinza como do Doppler colorido, utilizando-se o resultado da biópsia como padrão-ouro, bem como correlacionar os achados da ultra-sonografia e Doppler colorido com os resultados histopatológicos, segundo o escore de Gleason.

\section{MATERIAIS E MÉTODOS}

A população deste estudo consistiu de 88 pacientes consecutivos que foram referenciados ao nosso serviço para a realização de ultra-sonografia transretal da próstata, seguida de biópsia, no período de 28 de janeiro de 2003 a 17 de fevereiro de 2004, para elucidação diagnóstica devido a alteração no exame físico e/ou aumento dos níveis sanguíneos de PSA.

Os exames ultra-sonográficos e biópsias de todos os pacientes deste estudo foram realizados por, ou feitos sob supervisão de um único médico do serviço, para que as informações e a técnica de exame fossem as mais homogêneas possíveis.

Previamente à realização das biópsias, os pacientes foram orientados a realizar antibioticoprofilaxia com $400 \mathrm{mg}$ de norfloxacina, duas horas antes do procedimento, e utilizar um "fleet enema" para limpeza do reto uma hora antes do exame. $\mathrm{O}$ antibiótico era continuado por dois dias após a biópsia.

Todos os exames foram realizados em um equipamento ultra-sonográfico Aspen (Acuson Corporation; California, EUA), através de transdutor endocavitário EC7 de $7 \mathrm{MHz}$ tipo "end-fire".

O paciente era posicionado em decúbito lateral esquerdo e iniciava-se o exame no modo B, com a escala de cinza, de toda a glândula prostática e das vesículas seminais, fazendo-se varredura nos planos sa- gital e ortogonal (coronal oblíquo). Nesse momento, também era estimado o peso prostático, através da multiplicação das três dimensões da glândula (maiores eixos de comprimento, largura e altura) por um fator $\pi / 6$ (aproximadamente 0,52), assumindo-se que um mililitro de tecido prostático corresponde a, aproximadamente, um grama ${ }^{(6)}$.

Foram consideradas alterações suspeitas de câncer na ultra-sonografia achados do tipo nódulo hipoecóico em zona periférica, hipoecogenicidade difusa, perda da diferenciação entre zona periférica e a glândula interna, abaulamentos focais ou assimetria da zona periférica, irregularidades e interrupção da cápsula prostática ${ }^{(6,8,9)}$.

Após a varredura na escala de cinza, seguia-se o estudo com Doppler colorido. Para a realização deste estudo, a janela de cor era ajustada para cobrir toda a zona periférica e feixes neurovasculares, e a maior parte da glândula interna (o que eventualmente não era possível em glândulas de volume muito aumentado), a fim de permitir comparação do padrão de vascularização entre os dois lobos. Caso fosse encontrada alguma área de vascularização alterada ao Doppler colorido, a caixa de cor era reduzida de tamanho para aumentar a sensibilidade e permitir melhor avaliação da alteração focal.

O ganho do Doppler colorido foi ajustado para o ponto logo abaixo do limite de ruído. Foram usados parâmetros de baixa velocidade e alta sensibilidade. $O$ filtro foi ajustado para otimizar a visualização de pequenos vasos e baixo fluxo.

As alterações ao estudo com Doppler colorido foram divididas em assimetria na vascularização (associada ou não a lesão focal vista na escala de cinza), e aumento focal ou difuso da vascularização observada na zona periférica.

As biópsias foram realizadas por via transretal, orientadas pelo ultra-som, utilizando-se pistola de biópsia Pro Mag 2,2 (Medical Devices Technologies; Florida, EUA) com agulha de 18 Gauge.

Em cada zona periférica foram retirados de quatro a seis fragmentos aleatórios, além de biópsias dirigidas (um a dois fragmentos) em caso de alterações focais detectadas na escala de cinza e/ou ao Doppler colorido.
Os fragmentos obtidos foram colocados em frascos contendo formol a $10 \%$ e identificados quanto à sua localização (lateralidade). Fragmentos obtidos de biópsia dirigida foram colocados em frascos separados e identificados. Os fragmentos eram depois enviados para o serviço de patologia para análise.

A rotina do serviço de patologia para fragmentos prostáticos consiste na confecção de lâminas coradas pela hematoxilinaeosina e posterior análise. Para fim deste trabalho, os achados patológicos foram categorizados como benignos (normal, hiperplasia benigna, prostatite, infarto) e malignos (neoplasia intra-epitelial prostática, câncer). Nos espécimes com diagnóstico de câncer, o escore de Gleason foi calculado.

As dosagens de PSA foram feitas previamente ao exame, utilizando o "kit" policlonal, através do método de quimioluminescência, sendo considerados valores normais os menores de 4,00 ng/ml.

Para realizar os cálculos de sensibilidade, especificidade e valores preditivos positivo e negativo, foram definidos como casos verdadeiro-positivos aqueles com achado suspeito à ultra-sonografia e histopatologia positiva para câncer; como verdadeiro-negativos, na ausência de achados suspeitos à ultra-sonografia e com achado benigno à patologia; como falso-positivos, aqueles com achados suspeitos à ultra-sonografia e achados benignos no estudo patológico; e como falso-negativos, os casos sem achados suspeitos ao exame ultra-sonográfico, mas com diagnóstico de câncer à histopatologia.

A comparação de valores numéricos, como idade, valores de PSA, peso da glândula e escore de Gleason, foi feita utilizando-se o teste $t$ de Student para amostras não pareadas, sendo considerados significativos valores de $p$ menores que 0,05 .

\section{RESULTADOS}

Dos 88 pacientes estudados, quatro foram excluídos por apresentarem material insuficiente para análise histopatológica. Dessa forma, 84 pacientes foram investigados.

Nesses 84 pacientes, a biópsia foi positiva para adenocarcinoma em $31(36,9 \%)$ 
e negativa para adenocarcinoma em 54 $(63,1 \%)$. Não houve casos de neoplasia diferente do adenocarcinoma de próstata.

A idade média dos pacientes foi de 70,4 anos (variando de 46 a 94 anos), sendo de 68,9 anos (variando de 46 a 86 anos) nos pacientes com biópsia negativa e de 72 anos (variando de 56 a 94 anos) nos pacientes com biópsia positiva para adenocarcinoma. A diferença não foi estatisticamente significativa (valor de $p>0,05$ ).

O peso médio da próstata foi de $43,8 \mathrm{~g}$ nos pacientes sem neoplasia prostática e de 39,6 g nos pacientes com adenocarcinoma (diferença não significativa estatisticamente, valor de $p>0,05)$.

Quando os pacientes são divididos em grupos, de acordo com o peso prostático, em menores que $20 \mathrm{~g}$, entre $20 \mathrm{~g}$ e $50 \mathrm{~g}$ e maiores que $50 \mathrm{~g}$, observa-se que somente três $(5,7 \%)$ pacientes com diagnóstico benigno apresentaram próstata de volume normal (menor que $20 \mathrm{~g}$ ), enquanto os demais $50(94,3 \%)$ apresentaram próstata de dimensões aumentadas, sendo que, desses 50 pacientes, $16(30,2 \%)$ apresentaram peso prostático maior que $50 \mathrm{~g}$. Já no grupo com diagnóstico histológico de adenocarcinoma, cinco (16\%) pacientes apresentaram próstata de volume normal e somente $16 \%$ dos pacientes apresentaram próstata maior que $50 \mathrm{~g}$.

Os valores de PSA variaram de 0,65 ng/ $\mathrm{ml} \mathrm{a} 511 \mathrm{ng} / \mathrm{ml}$, sendo que somente nove pacientes apresentaram valores menores que $4 \mathrm{ng} / \mathrm{ml}$ (10,7\%). Os valores médios dos níveis de PSA foram de 7,6 ng/ml em pacientes com resultados benignos à biópsia, e de $48,1 \mathrm{ng} / \mathrm{ml}$ em pacientes com resultados positivos para câncer, tendo sido essa diferença estatisticamente significativa (valor de $p<0,05$ ). Não se encontrou nenhum caso de adenocarcinoma com valores de PSA menores que 4,0 ng/ml (sensibilidade de $100 \%$ ), porém a maioria dos pacientes com biópsia negativa para câncer (44 pacientes; 83\%) também apresentou valores maiores que 4,0 ng/ml (especificidade de 17\%).

$\mathrm{O}$ aspecto ultra-sonográfico foi considerado normal (Figura 1) em 38 (45,2\%) pacientes e suspeito em 46 pacientes $(54,8 \%)$. Dentre os achados suspeitos, o mais comum foi o nódulo hipoecóico da zona periférica (Figuras 2, 3 e 4), observado em 29 pacientes $(58,7 \%)$, ao passo que a hipoecogenicidade difusa da zona periférica com perda da diferenciação zonal entre a zona periférica e a glândula interna (Figuras 5 e 6) foi observada em 19 pacientes $(36,9 \%)$ com ultra-sonografia suspeita, havendo ainda dois pacientes $(4,4 \%)$ que apresentaram abaulamento isoecóico da zona periférica. Foram encontradas 57 alterações nos 46 pacientes com ultra-sonografia suspeita. Quatro pacientes apresentaram tanto área de hipoecogenicidade difusa quanto nódulo hipoecóico, sendo que em um deles havia dois nódulos além da área heterogênea. Cinco pacientes apresentaram dois nódulos hipoecóicos na zona periférica.

Correlacionando os achados ultra-sonográficos na escala de cinza com o resultado histopatológico, observou-se que 21 pacientes foram verdadeiro-positivos, 25 pacientes foram falso-positivos, 28 pacientes foram verdadeiro-negativos e dez pacientes foram falso-negativos (Quadro 1).
Figura 1. Aspecto normal da glândula prostática à ultra-sonografia. Observar a zona periférica homogênea e mais ecogênica do que a glândula central.
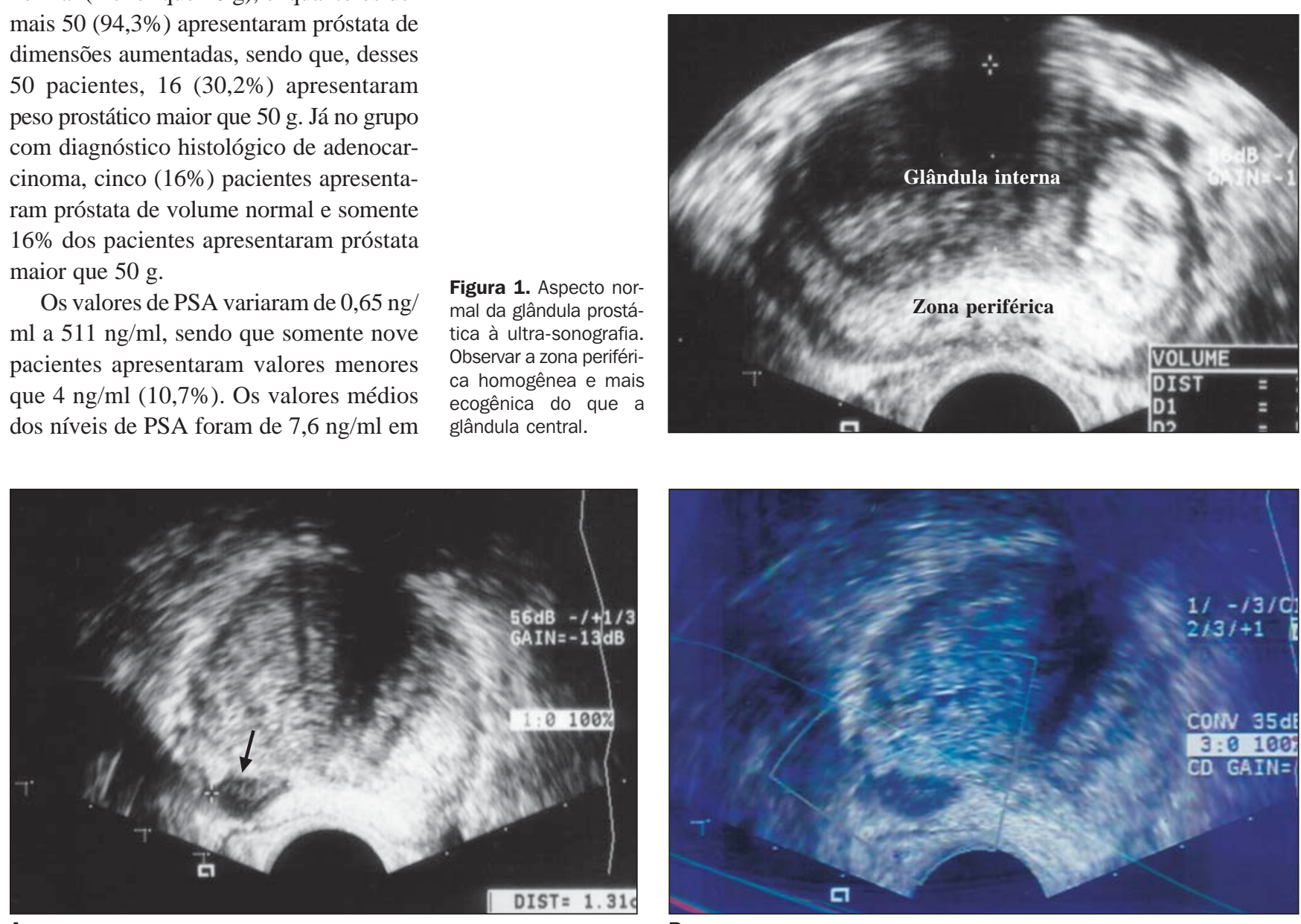

A

B

Figura 2. Nódulo hipoecóico na zona periférica do lobo direito (A). Ao Doppler colorido não se observa aumento da vascularização focal (B). À histopatologia observou-se área de infarto. 


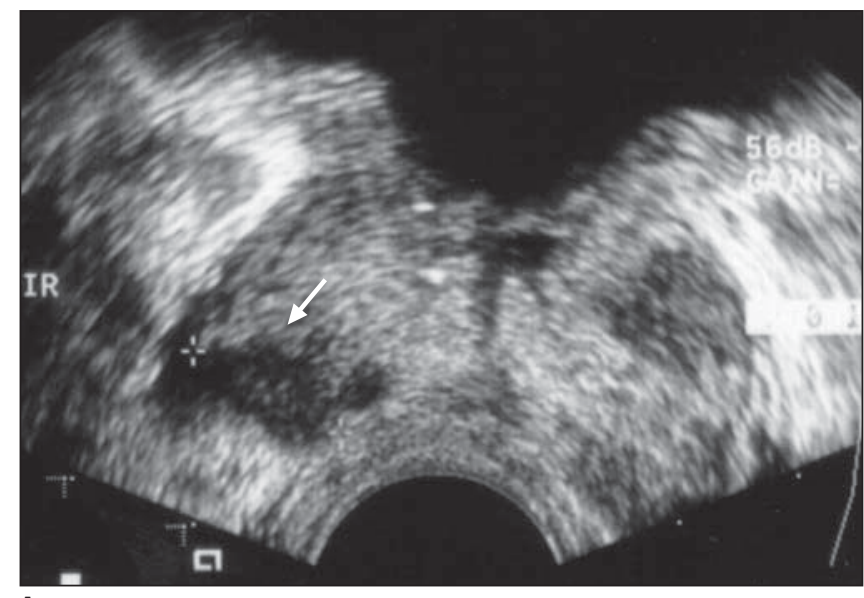

A

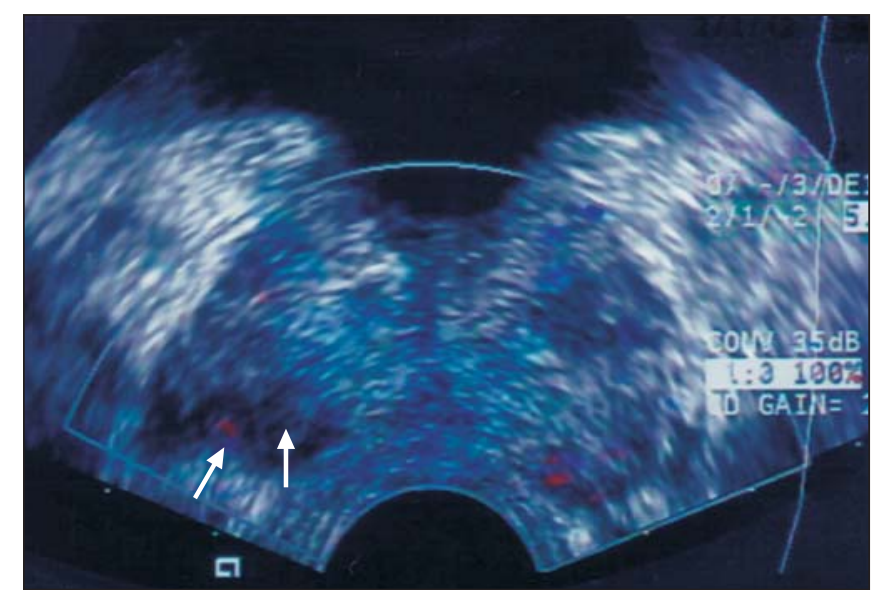

B

Figura 3. Nódulo hipoecóico na zona periférica direita (A). Ao Doppler colorido observa-se foco de hipervascularização no seu interior (B). A biópsia revelou adenocarcinoma Gleason $7(4+3)$.

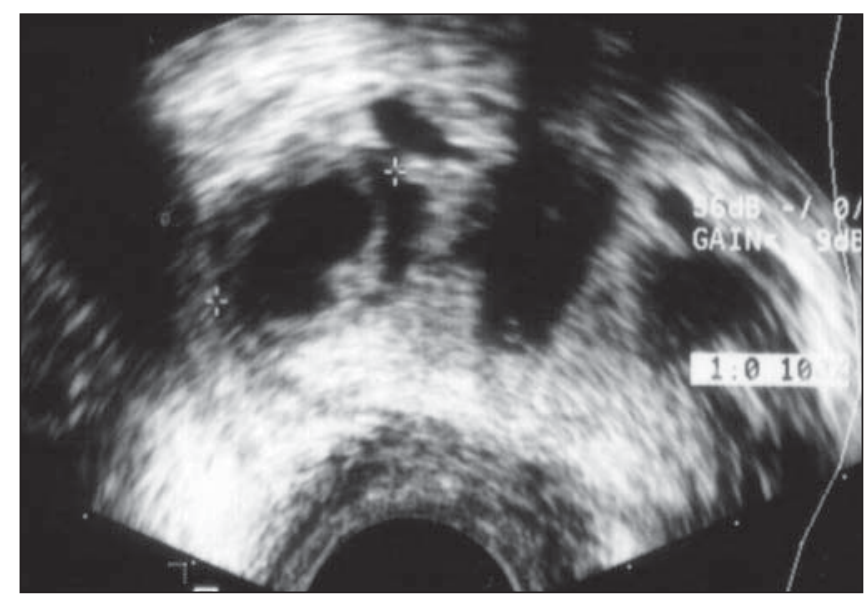

A

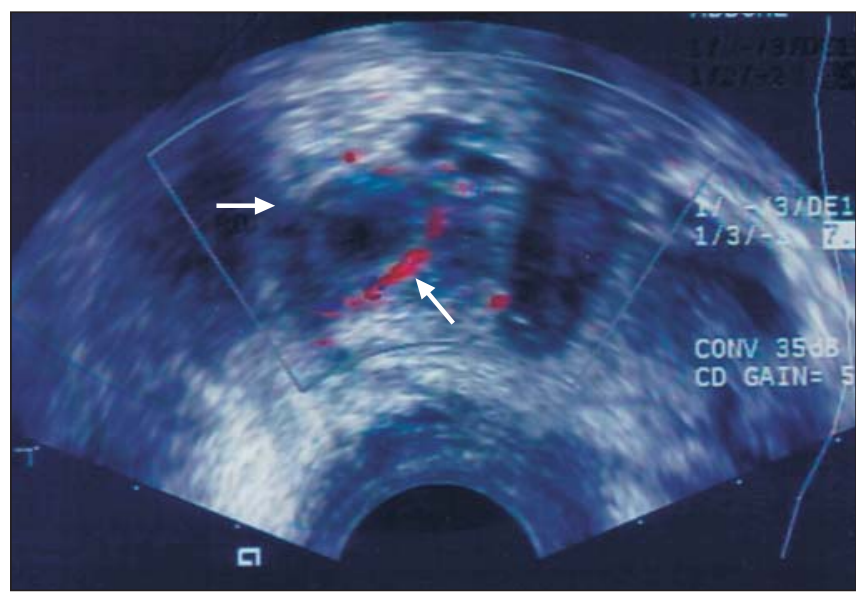

B

Figura 4. Nódulo hipoecóico na zona periférica direita, apresentando área cística de permeio (A). No Doppler colorido observa-se hipervascularização na periferia (parte sólida) do nódulo (B). A patologia demonstrou adenocarcinoma Gleason 7 (4+3) com infiltração perineural.

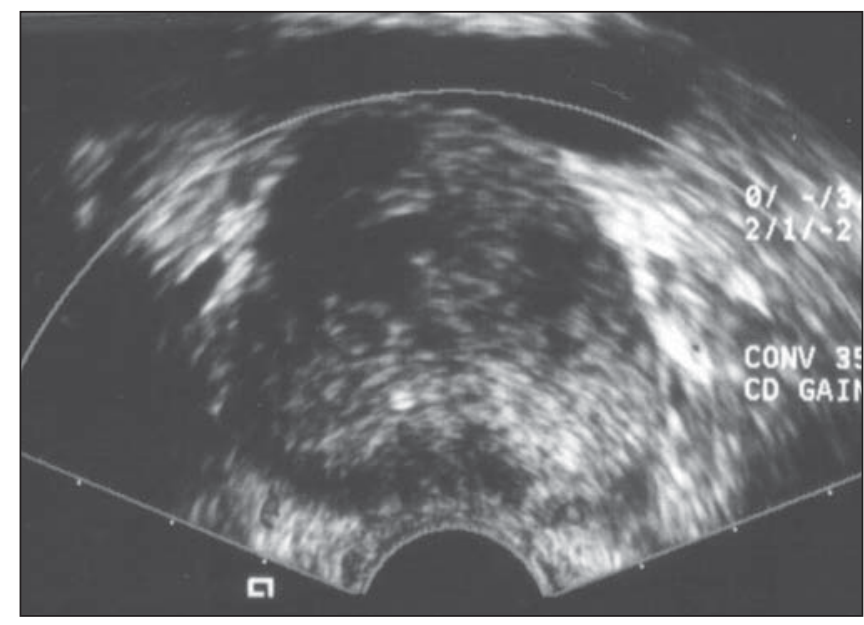

A

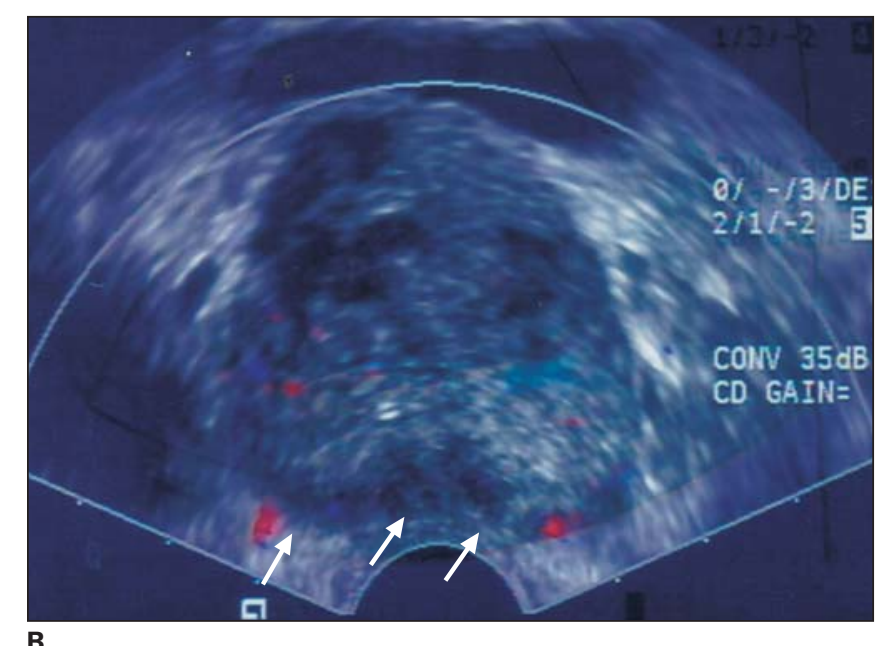

$\mathbf{B}$

Figura 5. Área de hipoecogenicidade difusa acometendo toda a zona periférica da próstata (A). Ao Doppler colorido (B) observa-se hipervascularização difusa da zona periférica. A biópsia evidenciou adenocarcinoma Gleason 6 bilateral. 
Figura 6. Hipoecogenicidade sem formar nódulos na zona periférica do lobo direito. A histopatologia foi benigna.

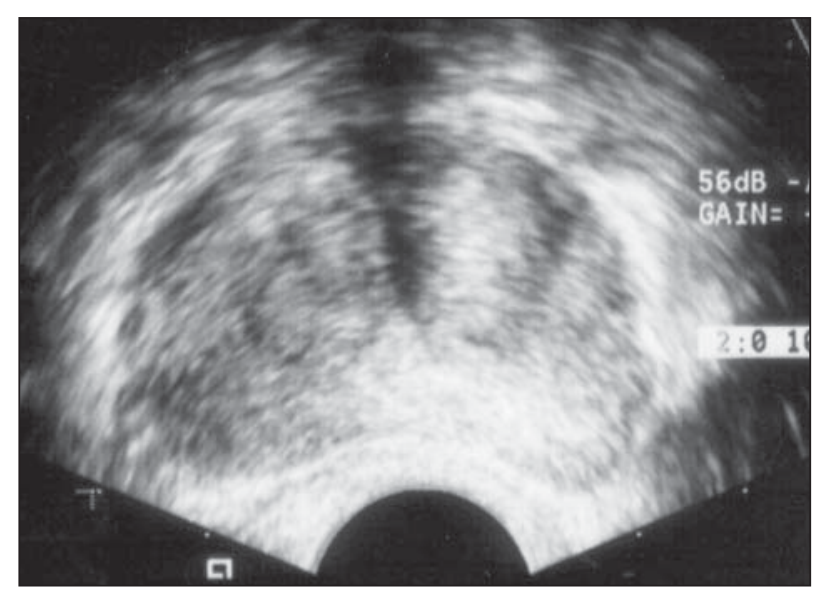

Quadro 1 Correlação dos achados ultra-sonográficos e histopatológicos.

\begin{tabular}{|l|c|c|c|}
\hline \multirow{2}{*}{ Ultra-sonografia } & \multicolumn{2}{|c|}{ Adenocarcinoma } & \\
\cline { 2 - 3 } & Positivo & Negativo & \multirow{2}{*}{ Total } \\
\hline Suspeita & 21 & 25 & 46 \\
Não suspeita & 10 & 28 & 38 \\
\hline Total & 31 & 53 & 84 \\
\hline
\end{tabular}

Portanto, para um achado suspeito à ultra-sonografia, obtivemos sensibilidade de $67,7 \%$, especificidade de $52,8 \%$ e valores preditivos positivo e negativo de $45,6 \%$ e $73,6 \%$, respectivamente.

Quando dividimos os dados pelos achados suspeitos, observamos que dos 29 pacientes com nódulos hipoecóicos na zona periférica, 12 tiveram biópsias positivas para adenocarcinoma, ou seja, valor preditivo positivo de $41,4 \%$. Já entre os pacientes com perda de diferenciação zonal, 11 dos 19 tiveram biópsias positivas (valor preditivo positivo de 57,9\%). Os dois pacientes que apresentaram abaulamento isoecóico tiveram biópsias negativas para adenocarcinoma (valor preditivo positivo de $0 \%$ ).

Em relação aos achados ao Doppler colorido, eles foram negativos em 55 pacientes, e positivos, ou seja, com algum aumento focal ou difuso do fluxo na glândula prostática, em 29 pacientes. Todas as alterações encontradas ao Doppler estavam relacionadas a alterações observadas à escala de cinza (Figuras 3B, 4B e 5B).

Correlacionando-se os achados ao Doppler colorido com a histopatologia, obtivemos 18 pacientes verdadeiro-positivos, 11
Quadro 2 Correlação dos achados ao Doppler colorido com a histopatologia.

\begin{tabular}{|l|c|c|c|}
\hline \multirow{2}{*}{ Doppler colorido } & \multicolumn{2}{|c|}{ Adenocarcinoma } & \\
\cline { 2 - 3 } & Positivo & Negativo & \multirow{2}{*}{ Total } \\
\hline Positivo & 18 & 11 & 29 \\
Negativo & 13 & 42 & 55 \\
\hline Total & 31 & 53 & 84 \\
\hline
\end{tabular}

pacientes falso-positivos, 42 pacientes verdadeiro-negativos e 13 pacientes falso-negativos (Quadro 2).

Dessa forma, levando-se em consideração apenas os achados ao Doppler colorido, os valores de sensibilidade, especificidade, valor preditivo positivo e valor pre$58,0 \%, 79,2 \%, 62,0 \%$ e $76,3 \%$.

Observa-se, então, que a adição do estudo do Doppler colorido ao exame ultrasonográfico de rotina levou a um aumento da especificidade (de $52,8 \%$ para $79,2 \%$ ) e do valor preditivo positivo (de $45,6 \%$ para $62,0 \%$ ) do exame; em contrapartida, houve redução da sensibilidade (de 67,7\% para $58,0 \%$ ).

Correlacionando-se os achados à escala de cinza com os achados do Doppler colorido, observamos que o aspecto mais freqüente foi a ultra-sonografia normal, em 38 pacientes, seguida do nódulo hipoecóico, visto em 29 pacientes, sendo que em 19 deles estava associado a alteração focal do fluxo ao Doppler.

A alteração hipoecóica difusa, com perda da diferenciação zonal, foi vista em 19 pacientes, sendo que em 12 deles também havia alteração dopplerfluxométrica. ditivo negativo foram, respectivamente, de
Em três pacientes, a presença de nódulo hipoecóico e heterogeneidade difusa coexistiram, embora em zonas periféricas distintas, sendo, em dois deles, ambos hipervasculares. Em um outro paciente coexistiram dois nódulos (um deles hipervascular), além de uma área heterogênea, hipoecóica, sem aumento da vascularização.

O escore de Gleason médio foi de 6,9, e o valor mais frequientemente encontrado foi $7(4+3)$, presente em 14 dos pacientes (45,2\% dos pacientes com biópsia positiva para adenocarcinoma).

Não houve diferença estatisticamente significativa entre os valores de Gleason dos pacientes com ultra-sonografia suspeita ou não à escala de cinza (médias de 6,71 e 6,67, respectivamente) ou ao Doppler colorido (médias de 7,1 e 6,6, respectivamente) (valores de $p>0,05)$. A diferença entre os valores médios do escore de Gleason foi significativa apenas quando se comparou o grupo com ultra-sonografia normal e os que apresentaram heterogeneidade difusa com hipervascularização (valor de $p<0,05$ ). Quando comparamos os grupos que apresentaram alterações no exame ultra-sonográfico, observamos que embora os pacientes que apresentaram alterações difusas com hipervascularização possuam valor médio do escore de Gleason mais elevado, essa diferença não é significativa do ponto de vista estatístico (valor de $p>0,05)$.

\section{DISCUSSÃO}

No estudo realizado, a prevalência do adenocarcinoma de próstata detectado à histopatologia, considerada aqui como padrão-ouro, foi de 39,3\%. Este valor está de acordo com trabalhos que apresentaram método de captação de pacientes semelhante ao realizado, em que a prevalência variou de $40 \%{ }^{(2)}$ a $47,5 \%{ }^{(10)}$.

$\mathrm{O}$ volume prostático foi maior, embora não estatisticamente significativo, nos pacientes com biópsias benignas, e este achado pode estar relacionado à maior prevalência de hiperplasia benigna, que tem sua prevalência aumentada com a idade ${ }^{(11)}$.

A dosagem sanguínea do PSA apresentou sensibilidade de $100 \%$, quando utilizado o valor de $4 \mathrm{ng} / \mathrm{ml}$ como valor de corte da normalidade. Não houve nenhum caso 
de malignidade em pacientes com valores abaixo do limite normal.

Este achado correlaciona-se parcialmente com dados da literatura, que afirmam que é rara a ocorrência de carcinoma prostático em pacientes com níveis normais de PSA. Em um estudo de Spencer et al..$^{(\mathbf{1 2})}$, com pacientes que possuíam valores de PSA normal, foi encontrada incidência de $19 \%$ de câncer prostático. No nosso estudo, a ausência de casos de câncer com PSA normal pode se dever ao fato do número menor de pacientes.

Por outro lado, este nível de corte de 4 $\mathrm{ng} / \mathrm{ml}$ para o valor do PSA apresentou especificidade de apenas $17 \%$, apesar de os valores médios entre os grupos de pacientes com câncer e com histopatologia benigna terem sido estatisticamente significativos. Essa sobreposição é observada porque tanto o parênquima prostático normal quanto o hiperplásico e o neoplásico produzem o PSA; dessa forma, pacientes com hiperplasia prostática podem apresentar-se com o PSA acima dos níveis normais $^{(5,13)}$.

A ultra-sonografia na escala de cinza demonstrou sensibilidade de $67,7 \%$, com valor preditivo positivo de $45,6 \%$, além de especificidade de $52,8 \%$. Esses valores estão dentro da faixa encontrada em vários estudos, em que os valores de sensibilidade variaram de $41 \%{ }^{(2)}$ a $96 \%{ }^{(10)}$, a especificidade variou de $27 \%^{(14)}$ a $81 \%^{(2)}$ e o valor preditivo positivo oscilou entre $45 \%{ }^{(15)}$ e $53 \%{ }^{(10)}$.

Já os achados ao Doppler colorido demonstraram sensibilidade de 58,0\%, valor preditivo positivo de $62,0 \%$ e especificidade de 79,2\%. Novamente, os valores se correlacionaram com os da literatura, em que a sensibilidade variou de $43,2 \%^{(2)}$ a $86,6 \%{ }^{(10)}$, o valor preditivo positivo variou de $40,8 \%^{(2)}$ a $77 \%^{(10)}$ e a especificidade variou de $38 \%{ }^{(15)}$ a $66,4 \%{ }^{(2)}$. Observa-se que, no presente estudo, a especificidade apresentou valores maiores que os da literatura, o que, na associação geral dos métodos, fez com que o Doppler colorido no trabalho atual tenha uma função adjuvante mais bem determinada.

Correlacionando-se os achados à escala de cinza com os achados do Doppler colorido, observamos que o aspecto mais frequientemente associado ao câncer prostá- tico foi o nódulo de zona periférica com hipervascularização focal associada, encontrado em 12 pacientes; somente um paciente com nódulo da zona periférica e adenocarcinoma à biópsia apresentou nódulo sem hipervascularização.

Esses achados estão de acordo com o achado histopatológico de que os tumores prostáticos geralmente se apresentam como áreas hipoecóicas nodulares, devido ao seu arranjo celular distinto da zona periférica normal, e que cerca de $76 \%$ dos cânceres nodulares são marcadamente hipoecói$\cos ^{(\mathbf{1 6})}$. De maneira semelhante, estudos mostram que os cânceres prostáticos apresentam maior densidade de microvasculatura, com vasos de diâmetro menor do que o observado em tecido normal (angiogênese tumoral) e ocupando área maior do tecido, o que se refletiria em hipervascularização ao Doppler ${ }^{(\mathbf{1 7})}$.

Por outro lado, 17 pacientes com biópsia benigna também apresentaram nódulo hipoecóico à ultra-sonografia, sendo que em oito dos casos o nódulo era hipervascular ao Doppler colorido.

Três desses pacientes com nódulo hipervascular tiveram diagnóstico de prostatite aguda e/ou crônica e os demais pacientes tiveram diagnóstico de hiperplasia benigna prostática ou histologia normal. Já entre os pacientes com nódulo sem aumento de vascularização, houve um caso de infarto, um de hiperplasia nodular e um de prostatite crônica; os demais casos tiveram resultado normal ou de hiperplasia prostática benigna.

A lesão hipoecóica à ultra-sonografia não é específica do adenocarcinoma da próstata, existindo inúmeras lesões benignas, como hiperplasia focal, infartos, prostatite aguda ou crônica, que podem se apresentar com este aspecto à ecografia. Rifkin e Choi ${ }^{(8)}$, estudando lesões prostáticas periféricas, observaram que apenas cerca de $20 \%$ das lesões hipoecóicas foram malignas ao estudo anatomopatológico.

A hipervascularização em uma lesão benigna também pode ser explicada pela histopatologia, uma vez que se observa aumento do número de vasos, porém de dimensões normais ou aumentados (não vasos semelhantes aos vistos na angiogênese tumoral), o que aumenta o percentual de área de lesão ocupada por vasos e pode se refletir, no Doppler colorido, em hipervascularização $^{(17)}$.

Em relação ao achado de heterogeneidade difusa, dos 11 casos com diagnóstico de adenocarcinoma, nove apresentaram aumento difuso da vascularização na região, enquanto dois pacientes apresentaram fluxo normal na glândula prostática. Em contrapartida, dos nove pacientes com biópsia benigna com este mesmo achado, somente três apresentaram hipervascularização, tendo sido o diagnóstico prostatite crônica e hiperplasia benigna. Os demais seis pacientes com heterogeneidade difusa sem hipervascularização tiveram diagnóstico histológico de normalidade/hiperplasia benigna.

A alteração difusa de perda da diferenciação zonal entre as zonas central e periférica foi descrita por Ezz el Din e de la Rosette $^{(\mathbf{6})}$ como um achado específico para o câncer de próstata.

Neste trabalho, o valor preditivo positivo da alteração difusa na ultra-sonografia para o diagnóstico de câncer foi de $57,9 \%$. Quando associamos a positividade ao Doppler colorido à alteração na escala de cinza, o valor preditivo positivo aumenta para $75 \%$. Em contrapartida, o valor preditivo positivo para uma lesão focal, nodular, hipoecóica na zona periférica foi, neste trabalho, de 41,4\%, aumentando para $57,9 \%$ quando tal alteração também era positiva ao Doppler colorido; a alteração difusa foi um sinal ultra-sonográfico mais específico de carcinoma prostático do que o nódulo hipoecóico.

Nos dois casos de abaulamento isoecóico, o estudo com Doppler colorido foi negativo e a biópsia, benigna. Isto provavelmente indica que o achado correspondia apenas a assimetria por hiperplasia benigna, ou nódulos hiperplásicos que promoviam abaulamentos na zona periférica, tornando-a visualmente assimétrica.

Em dez dos 31 pacientes, o câncer da próstata não foi evidenciado por nenhum dos dois métodos, só tendo sido diagnosticado por meio da biópsia aleatória. $\mathrm{Ou}$ seja, 32,2\% dos adenocarcinomas do atual estudo se apresentaram isoecóicos ao parênquima prostático adjacente, o que está de acordo com os achados de Shinohara et $a l .{ }^{(9)}$, que encontraram $39 \%$ de tumores não visualizados ao ultra-som. 
Nestes pacientes, o diagnóstico de câncer foi visto em menos da metade dos fragmentos, e em todos eles o acometimento foi unilateral, não havendo nenhum caso de carcinoma prostático difuso. Além disso, em nenhum destes casos houve descrição de invasão perineural.

Por outro lado, o escore de Gleason médio dos cânceres isoecóicos foi de 6,67, tendo um paciente o escore de 8 ; porém, este só possuía um fragmento positivo para adenocarcinoma, ou seja, os cânceres "invisíveis" ao ultra-som também são clinicamente significativos (possuem Gleason maior que 6), embora pareçam apresentar extensão menor.

Esse achado está de acordo com o trabalho de Lee et al. ${ }^{(3)}$, que não encontraram diferença entre os escores de Gleason de tumores palpáveis e impalpáveis, e o de Slonim et al. ${ }^{(\mathbf{1 8})}$, que acharam que a biópsia aleatória aumentou em $28 \%$ a detecção de câncer, e que os cânceres encontrados por esse método eram clinicamente significativos. Observa-se também correlação parcial com o trabalho de Shinohara et al..$^{(\mathbf{9})}$, que acharam diferença significativa entre os tumores hipoecóicos e os isoecóicos, sendo os primeiros menos diferenciados, que pode ser parcialmente explicada pelo tamanho do foco tumoral, uma vez que Shinohara et al. ${ }^{(\mathbf{9})}$ observaram que somente $18 \%$ dos tumores com menos de $10 \mathrm{~mm}$ foram identificados à ultra-sonografia.

Os escores de Gleason de lesões nodulares ou difusas (perda da diferenciação) e de lesões com ou sem aumento da vascularização ao Doppler colorido não foram estatisticamente significativos entre si, embora as lesões hipervasculares apresentem valores médios maiores do que as lesões sem alteração ao Doppler colorido. Porém, quando comparado com o grupo de pacientes no qual o diagnóstico foi feito somente pela biópsia (ultra-sonografia normal), observou-se diferença significativa estatisticamente, refletindo uma maior agressividade dos tumores nesses casos. De fato, o aumento da microangiogênese observada nos casos de tumor ${ }^{(\mathbf{1 7 )}}$ e a relação explicada por Shinohara et al. ${ }^{(9)}$ da agressividade tumoral podem justificar essa diferença, embora não haja relato na literatura da relação da positividade ao Doppler colorido com um maior grau na escala de Gleason.
Não houve nenhum caso de complicação importante pelo procedimento da biópsia e não houve queixas álgicas significativas que impossibilitassem o procedimento. A técnica de biópsias realizadas no atual estudo consistiu em biópsias dirigidas a lesões evidenciadas ao exame, além de, em média, oito a dez fragmentos de biópsias aleatórias, bilateralmente.

Segundo Hodge et al. ${ }^{(19)}$, o uso de biópsias aleatórias pode aumentar em até $20 \%$ a taxa de detecção do câncer prostático. Slonim et al. ${ }^{(\mathbf{1 8})}$ também observaram aumento da taxa de detecção de câncer de $28 \%$ com o uso de biópsias aleatórias. No presente estudo, 32,2\% dos resultados de malignidade foram diagnosticados apenas através da biópsia aleatória.

De qualquer forma, a técnica de biópsia ainda não é um consenso. Inicialmente, Hodge et al. ${ }^{\left({ }^{(19)}\right.}$ estabeleceram o uso de seis fragmentos (três em cada lobo), no ápice, porção média e base prostática. Posteriormente, vários autores questionaram sua capacidade de detecção, como Presti et $a l .{ }^{(20)}$ e Ravery et al. ${ }^{\text {(21) }}$, afirmando que era necessário um maior número de fragmentos biopsiados, ao passo que outros autores, como Naughton et al. ${ }^{(\mathbf{2 2})}$, não observaram vantagem significativa no aumento do número de fragmentos para a detecção do câncer.

A Sociedade Brasileira de Urologia, em trabalho conjunto com o Colégio Brasileiro de Radiologia, também preconizam o protocolo de biópsia sextante estendida (com 12 fragmentos) como o padrão de biópsias transretais, devido à maior capacidade diagnóstica usando-se este protocolo ${ }^{(23)}$.

Analisando os dados do presente estudo, no contexto do diagnóstico do câncer da próstata, verificamos a limitação da ultra-sonografia para tal. Uma vez que o rastreamento inicial dos pacientes é feito pelos níveis de PSA e toque retal, a ultrasonografia deveria ter a função de tentar diminuir o número de biópsias negativas (desnecessárias), sem prejudicar a capacidade de detecção do câncer. No entanto, o caráter inespecífico do aumento do PSA acima de $4 \mathrm{ng} / \mathrm{ml}$, associado a sensibilidade e especificidade baixas da ultra-sonografia, mesmo com a adição do Doppler colorido, prejudica sua utilização como método de rastreio.
De qualquer forma, a ultra-sonografia é útil, pois permite a realização, além de biópsias aleatórias, de biópsias direcionadas a alterações ecográficas, que possuem maior valor preditivo positivo para carcinoma.

\section{REFERÊNCIAS}

1. INCA - Instituto Nacional do Câncer. Câncer de próstata/Epidemiologia. Instituto Nacional do Câncer, 2003. Disponível em: URL:http//:www. inca.gov.br

2. Kuligowska E, Barish MA, Fenlon HM, Blake M. Predictors of prostate carcinoma: accuracy of gray scale and color Doppler US and serum markers. Radiology 2001;220:757-764.

3. Lee F Jr, Bronson JP, Lee F, et al. Nonpalpable cancer of the prostate: assessment with transrectal US. Radiology 1991;178:197-199.

4. Littrup PJ, Kane RA, Williams CR, et al. Determination of prostate volume with transrectal US for cancer screening. Part I: Comparison with prostate-specific antigen assays. Radiology 1991; 178:537-542.

5. Stamey TA, Kabalin JH. Prostate specific antigen in the diagnosis and treatment of adenocarcinoma of the prostate. I. Untreated patients. J Urol 1989; 141:1070-1075.

6. Ezz el Din K, de la Rosette JJMCH. Transrectal ultrasonography of the prostate. Br J Urol 1996; 78:2-9.

7. Rifkin MD, Dähnert W, Kurtz AB. State of the art: Endorectal sonography of the prostate gland. AJR Am J Roentgenol 1990;154:691-700.

8. Rifkin MD, Choi H. Implications of small, peripheral hypoechoic lesions in endorectal US of the prostate. Radiology 1988;166:619-622.

9. Shinohara K, Wheeler TM, Scardino PT. The appearance of prostate cancer on transrectal ultrasonography: correlation of imaging and pathological examinations. J Urol 1989;142:76-82.

10. Kelly IMG, Lees WR, Rickards D. Prostate cancer and the role of color Doppler US. Radiology 1993;189:153-156.

11. Sagalowsky AI, Wilson JD. Hyperplasia and carcinoma of the prostate. In: Fauci AS, Braunwald E, Isselbacher KJ, editors. Harrison's Principles of internal medicine. 14th ed. New York, NY: McGraw-Hill 1998;596-602.

12. Spencer JA, Alexander AA, Gomella L, Matteucci T, Goldberg BB. Clinical and US findings in prostate cancer: patients with normal prostatespecific antigen levels. Radiology 1993;189: 389-393.

13. Stamey TA, Kabalin JH, McNeal JE, et al. Prostate specific antigen in the diagnosis and treatment of adenocarcinoma of the prostate. II. Radical prostatectomy treated patients. J Urol 1989; 141:1076-1083.

14. Rubens DJ, Gottlieb RH, Maldonado Jr CE, Frank IN. Clinical evaluation of prostate biopsy parameters: gland volume and elevated prostate-specific antigen level. Radiology 1996;199:159-163.

15. Lavoipierre AM, Snow RM, Frydenberg M, et al. Prostate cancer: role of color Doppler imaging in transrectal sonography. AJR Am J Roentgenol 1998;171:205-210.

16. Lee F, Torp-Pederssen S, Littrup PJ, et al. Hypoechoic lesions of the prostate: clinical relevance 
of tumor size, digital rectal examination, and prostate-specific antigen. Radiology 1989;170: 29-32.

17. Louvar E, Littrup PJ, Goldstein A, Yu L, Sakr W, Grignon D. Correlation of color Doppler flow in the prostate with tissue microvascularity. Cancer 1998;83:135-140.

18. Slonim SM, Cuttino JT Jr, Johnson CJ, et al. Diagnosis of prostatic carcinoma: value of random transrectal sonographically guided biopsies. AJR Am J Roentgenol 1993;161:1003-1006.
19. Hogde KK, McNeal JE, Terris MK, Stamey TA. Random systematic versus directed ultrasound guided transrectal core biopsies of the prostate. J Urol 1989;142:71-75.

20. Presti JC Jr, Chang JJ, Bhargava V, Shinohara K. The optimal systematic prostate biopsy scheme should include 8 rather 6 biopsies: results of a prospective clinical trial. J Urol 2000;163:163167.

21. Ravery V, Goldblatt L, Royer B, Blanc E, Toublanc M, Boccon-Gibod L. Extensive biopsy protocol improves the detection rate of prostate cancer. J Urol 2000;164:393-396.

22. Naughton CK, Miller DC, Mager DE, Ornstein $\mathrm{DK}$, Catalona WJ. A prospective randomized trial comparing 6 versus 12 prostate biopsy cores: impact on cancer detection. J Urol 2000;164: 388-392.

23. Cury J, Prando A, Billis A, et al. Biópsia prostática - padronização. Documento de consenso. Sociedade Brasileira de Urologia, biênio 2002/ 2003. 